\title{
Severe Abdominal Pain as the First Manifestation of Rabies
}

\author{
Jamshid Ayatollahi ${ }^{1}$; Mohammad Reza Sharifi ${ }^{1}$; Seyed Hossein Shahcheraghi ${ }^{1,}$ \\ ${ }^{1}$ Infectious and Tropical Diseases Research Center, Shahid Sadoughi University of Medical Sciences, Yazd, IR Iran \\ ${ }^{*}$ Corresponding author: Seyed Hossein Shahcheraghi, Infectious and Tropical Diseases Research Center, Shahid Sadoughi University of Medical Sciences, Yazd, IR Iran. Tel: +98- \\ 9132531389, Fax:+98-3518224100, E-mail: shahcheraghih@gmail.com
}

Received: April 20, 2013; Revised: June 6, 2013; Accepted: July 7, 2013

\begin{abstract}
Introduction: Rabies is an acute fatal viral disease that is generally transmitted from animals to humans following wild and domestic animal bites. The rabies virus enters the body from the area where the individual is bitten, and then the virus moves towards the brain and involves the nerves.

Case Presentation: During the years 2001-2011, there have been 73 reported rabies cases. About 50,000 reported human deaths are annually due to rabies. The actual number of human deaths due to rabies in Asia especially India, Pakistan and Bangladesh are more than these numbers, since there is no advanced surveillance system for disease control to determine the actual number of infected and fatal human cases. According to the World Health Organization (WHO) reports, more than 10 million people who are bitten by animals are annually treated by prophylactic treatment regimens for rabies, worldwide.

Conclusions: This paper reports on a case of human rabies with the first disease manifestation (severe abdominal pain). The patient reported extensive biting on his left leg by a dog. He had a slight fever of $38.1^{\circ} \mathrm{C}$. It has been recommended that a careful history should be taken from patients for diagnosis of rabies disease. A complete history should be taken from patients for diagnosis of disease, because rabies could be wrong with various diseases with atypical symptoms. because various diseases with atypical symptoms or long incubation periods can visit.
\end{abstract}

Keywords: Rabies; Humans; Iran

\section{Introduction}

Zoonosis or common diseases between humans and animals can be transmitted from both domesticated and wild animals to individuals. The transmission of common diseases is related to many factors including: an available agent, the amount of exposure, mechanisms related to transferring and host sensitivity. These factors have principal roles in the epidemiology of fatal viral diseases in humans. The rabies virus (genus Lyssavirus, family Rhabdoviridae) is present in the saliva of infected mammals and is most commonly transmitted via a biting incident (1-4). The present study was performed to evaluate a case of human rabies with severe abdominal pain from Iran.

Rabies is an encephalitis agent in mammals, which causes a fatal viral zoonotic disease. It has been suggested that there are approximately 55000 victims per year of whom $30-50 \%$ are below 15 years of age. Nevertheless rabies is commonly diagnosed after the onset of neurological complaints and controlled by wound care, administration of rabies immune globulin (RIG) and vaccine, which are almost 100\% successful in inhibiting human rabies following disclosure. The rabies virus is present in the saliva of unhealthy mammals and is normally transferred via a biting incident (5).

Non-bite sources of rabies transmission are including: unhealthy mucous membranes with other pos- sibly infectious components (such as tracheal, nasal secretions and brain tissues, cerebrospinal fluid and tears), scratches, corneal transplantation or disclosure of membranes to aerosols. Unhealthy mucous membranes with other possibly infectious components (such as tracheal, nasal secretions and brain tissues, cerebrospinal fluid and tears), scratches, corneal transplantation or disclosure of membranes to aerosols have been reported as non-bite sources of rabies transmission (6).

The first signs of rabies disease in humans include: a common feeling of illness, pain at the bite site, nausea, vomiting, depression and headache. This virus begins to reproduce and multiplies in the brain or spinal cord; neurological signs appear, including anxiety, stun, hallucinations, production of abundant saliva, high-level of agitation, insomnia, paralysis of lower legs, fidgetiness, voice box spasms and problems swallowing due to a painful throat. In Iran, the Ministry of Health and Medical Education has established the WHO Collaborating Center for Reference and Research on Rabies, as the only testing laboratory in Iran, to follow rabies cases. In Asia, most mortality cases of human rabies were reported from underdeveloped countries such as India, Pakistan and Bangladesh which have large populations

Copyright (C 2014,Ahvaz Jundishapur University of Medical Sciences; Published by Kowsar Corp. This is an open-access article distributed under the terms of the Creative Commons Attribution License, which permits unrestricted use, distribution, and reproduction in any medium, provided the original work is properly cited. 
and no specific strategies for controlling rabies (7).

\section{Case Presentation}

On the 2nd of March 2013, a 26-year-old man resident of Rafsanjan (Kerman province, south-eastern, Iran) was admitted to Shahid Sadoughi Hospital of Yazd, Iran with symptoms of severe abdominal pain. The patient reported extensive biting on his left leg by a dog showing marked aggressiveness, on the 22nd of September 2012, eight months before symptomatic disease onset. The dog had bitten four people who had received rabies immune globulin (RIG) and vaccine while our study patient had not received this treatment. The dog died three days after the biting incident. On admission, our study patient stayed at the surgery unit for one day because of his severe abdominal pain followed by two days at the neurology unit because of behavioral changes. After consultation, he was transferred to the infectious diseases ward.

On the first day of hospital admission, the patient had malaise, diplopia, headache, depression, vomiting and severe abdominal pain in the hypogastria. He also had a five-year history of consumption of narcotic substances (opium) but had quit use since six months ago. Complete blood count revealed a normal count of leucocytes $(9200 / \mu \mathrm{L})$. Blood had a slight increase of glucose (111 mg/ dL). Wright and Widal tests were negative. Cerebrospinal fluid (CSF) was analyzed and showed 100\% lymphocytes, two white blood cells (WBC), 159 red blood cells (RBC) and protein $40 \mathrm{mg} / \mathrm{dL}$.

On the 3rd and 4th of March 2013, the patient had a slight fever $\left(38.1^{\circ} \mathrm{C}\right)$, malaise, headache, abdominal pain and abnormal behavior. Finally, the patient died on the 4th of March 2013. Cerebrospinal fluid sample was submitted to the Pasteur Institute of Tehran, Iran. The polymerase chain reaction (PCR) test was performed. This test was positive and proved rabies disease.

\section{Discussion}

Our patient was admitted with symptoms of severe abdominal pain, as the first and most severe manifestation. Therefore, it is necessary to obtain information from patients for diagnosis of disease, because rabies could be wrong with various diseases with atypical symptoms. because various diseases with atypical symptoms or long incubation periods can visit.

One of the most important diseases in developing countries, especially in poor rural communities, is rabies viral disease. Despite the production of several secure and effective human vaccines against the disease virus, virus victims continue to grow in many countries. The bite of a rabid animal is the main agent of virus transmission. Also, transmission can be via the saliva that is the major source of virus spread (5). Rabies disease is endemic in the wild life of our country, therefore domestic animals are frequently infected while, enhancing cases of animal bite and scattering of disease virus in most provinces of Iran, declare a need to pay more attention to controlling this disease (8).

In a study in Kerman province of Iran, 10 individuals were infected with rabies disease, two of them being females and eight males, and half of them were attacked by dogs. The incubation period was 54 days (9). A fatal case of human rabies was an Indian man in his forties, who had been a resident of Italy for 10 years. He was admitted with fever $\left(40.4^{\circ} \mathrm{C}\right)$, malaise, headache, diplopia, unilateral ptosis (left eye), whole body paraesthesia, ataxia, myalgia and flaccid paresis of the arms, especially of the left arm. His behavior appeared abnormal, with signs of anxiety and agitation (the incubation period was one month). Finally, the patient died (10).

Another study, reported a 27-year-old male with hypochondriasis. This patient had contracted rabies. He refused to respond correctly to several questions in the hospitalization time. The patient had severe rabidity and bitted himself and attacked individuals by biting them. He was been bitten by a dog (the incubation period was eight years). He had a common feeling of sickness, depression, and pain at the bite site, nausea and headache. Finally, the patient died (7).

The difference of our patient with other reported cases is the length of the incubation period (eight months). The first manifestation of the disease is severe abdominal pain.

\section{Acknowledgements}

We would like to thank the staff of the Infectious and Tropical Diseases Research Center of Yazd Shahid Sadoughi University of Medical Sciences and the Pasteur Institute of Iran for their cooperation with this study.

\section{Authors' Contributions}

Dr. Jamshid Ayatollahi developed the original idea and the protocol. Dr. Mohammad Reza Sharifi contributed to the development. Seyed Hossein Shahcheraghi prepared the manuscript.

\section{Funding/Support}

This study was financially supported by the Infectious and Tropical Diseases Research Center, Shahid Sadoughi University of Medical Sciences, Yazd, IR Iran.

\section{References}

1. Zandi F, Eslami N, Soheili M, Fayaz A, Gholami A, Vaziri B. Proteomics analysis of BHK-21 cells infected with a fixed strain of rabies virus. Proteomics. 2009;9(9):2399-407.

2. Woldehiwet Z. Clinical laboratory advances in the detection of rabies virus. Clin Chim Acta. 2005;351(1-2):49-63.

3. Kuzmin IV, Hughes GJ, Botvinkin AD, Gribencha SG, Rupprecht CE. Arctic and Arctic-like rabies viruses: distribution, phylogeny and evolutionary history. Epidemiol Infect. 2008;136(4):509-19.

4. Vazirianzadeh B, Rahdar M. Correct Identification of Animal Host Species Is Important in the Diagnosis of Zoonotic Diseases. Jundishapur J Microbiol. 2013;6(2):97-9.

5. Lafon M. Rabies virus receptors. J Neurovirol. 2005;11(1):82-7.

6. Malerczyk C, Detora L, Gniel D. Imported human rabies cases in 


\section{Ayatollahi J et al.}

europe, the United States, and Japan, 1990 to 2010. J Travel Med. 2011;18(6):402-7.

7. Seimenis A. The rabies situation in the Middle East. Dev Biol (Basel). 2008;131:43-53.

8. Hassanzadeh SM, Zavareh A, Shokrgozar MA, Ramezani A, Fayaz A. High vero cell density and rabies virus proliferation on fibracel disks versus cytodex-1 in spinner flask. PakJ Biol Sci. 2011;14(7):441-8.
9. Hatami H. History of Rabies in Traditional Medicine's Resources and Iranian Research Studies: On the Cccasion of the World Rabies Day (September 28, 2012). Int J Prev Med. 2012;3(9):593-5.

10. De Benedictis P, Perboni G, Gentili C, Gaetti L, Zaffanella F, Mutinelli F, et al. Fatal case of human rabies imported to Italy from India highlights the importance of adequate post-exposure prophylaxis, October 2011. Euro Surveill. 2012;17(19). 